\title{
Biologia de Agistemus floridanus Gonzalez (Acari, Stigmaeidae)
}

\author{
Noeli Juarez Ferla ${ }^{1} \&$ Gilberto José de Moraes ${ }^{2}$
}

\author{
${ }^{1}$ Museu de Ciências Naturais, Centro Universitário UNIVATES. 95900-000 Lajeado, Rio Grande do Sul, Brasil. E-mail: \\ njferla@fates.tche.br \\ ${ }_{2}^{2}$ Departamento de Entomologia, Fitopatologia e Zoologia Agrícola, Escola Superior de Agricultura Luiz de Queiroz. 13418 - \\ 900 Piracicaba, São Paulo, Brasil. Bolsista do CNPq. E-mail: gjmoraes@carpa.ciagri.usp.br
}

\begin{abstract}
Biology of Agistemus floridanus Gonzalez (Acari, Stigmaeidae). The rubber tree (Hevea brasiliensis Muell. Arg.) (Euphorbiaceae) is attacked by various species of phytophagous mites in Brazil. Studies conducted in the State of Mato Grosso showed the presence of Agistemus floridanus Gonzalez, 1965 (Stigmaeidae) on rubber trees, associated with the mite Tenuipalpus heveae Baker, 1945. The aim of this work was to study the biology of that predator in the laboratory, when fed with $T$. heveae as prey. The study was started with 22 eggs, which resulted in 15 females reaching adult hood. To determine the effect of mating on oviposition, 30 females were used; half of those were maintained isolated and the remaining were maintained then with males during the whole adult stage. The stage of egg was the longest, with a duration of more than 4.0 days. The total duration of the immature phase was 10.2 days. Each female oviposited an average of 38.4 eggs, with a daily oviposition rate of 2.3 eggs per female. The duration of each adult phase, the daily oviposition rate and the longevity were different between the mated females and non-mated females. All eggs produced by non-mated originated males, characterizing, in this way, the development through arrhenothokous parthenogenesis. The results showed that A. floridanus has an innate increase capacity of 13.2 times in each generation, that the average duration of one generation is 19.2 days, that the intrinsic rate of increase $(\mathrm{rm})$ is about 0.16 female per female per day and that the number of females added daily to the population is 1.1.
\end{abstract}

KEY WORDS: Acari, biological control, predator, rubber tree.

A seringueira (Hevea brasiliensis Muell. Arg.) (Euphorbiaceae) é hospedeira de várias espécies de ácaros fitófagos nas diversas regiões onde é cultivada no Brasil. Espécies de Phytoseiidae e Stigmaeidae são os ácaros predadores mais comuns na seringueira em associação com ácaros fitófagos (Feres 2000, Ferla \& Moraes 2002).

As espécies de Stigmaeidae encontradas em seringueira nos Estados do Mato Grosso e São Paulo pertencem aos gêneros Agistemus Summers, 1960 e Zetzellia Oudemans, 1927. Espécies destes gêneros são geralmente conhecidas como predadoras ativas de ovos de tetraniquídeos (Ehara 1985, Gerson \& SMiley 1990, Laing \& KNOp 1982, McMurtry et al. 1970).

Tenuipalpus heveae Baker, 1945 é um tenuipalpídeo presente na cultura da seringueira, sendo encontrado mais comumente na face abaxial dos folíolos. Entretanto, quando em grandes populações, pode ser observado na face adaxial (FerEs 2000, Ferla \& Moraes 2002). Causa amarelecimento e contribui para a queda prematura das folhas.

Agistemus floridanus Gonzalez, 1965 é um predador da família Stigmaeidae encontrado em altas populações associado a T. heveae e Calacarus heveae Feres, 1992 em seringueira no
Estado do Mato Grosso (Ferla \& Moraes 2002). Nenhum estudo foi realizado até momento para conhecer a tabela de vida e a fertilidade deste predador. O conhecimento de predadores com capacidade de controle de $T$. heveae dará suporte ao estabelecimento de programas de manejo que enfatizem o uso daqueles nesta cultura.

O objetivo deste trabalho foi obter informações sobre a biologia de $A$. floridanus quando alimentado com $T$. heveae.

\section{MATERIAL E MÉTODOS}

Este trabalho foi realizado nos laboratórios da Escola Superior de Agricultura "Luiz de Queiroz" (ESALQ/USP), em Piracicaba, São Paulo, utilizando-se ácaros provenientes de colônias de laboratório iniciadas com espécimes coletados no campo há cerca de dois meses. Os ácaros foram retirados de folhas de seringueira provenientes das Plantações Edouard Michelin Ltda., no município de Itiquira, Estado do Mato Grosso.

A colônia de $A$. floridanus foi mantida em unidades de criação formadas por folíolos de seringueira destacados, colo-

Revista Brasileira de Zoologia 20 (2): 261-264, junho 2003 
cados sobre esponja umedecida no interior de uma bandeja plástica. Para que os ácaros permanecessem na penumbra, as bandejas foram mantidas parcialmente cobertas. Os ácaros foram alimentados com uma mistura de diferentes estádios de Oligonychus gossypii (Zacher, 1921) e T. heveae, transferindo-se os predadores semanalmente para novas unidades de criação.

\section{Biologia de Agistemus floridanus}

Quadrados de $3 \mathrm{~cm}^{2}$ foram recortados de folíolos de seringueira e colocados com a face adaxial para baixo sobre esponja umedecida em uma bandeja de alumínio. As margens dos quadrados foram cobertas por uma faixa de algodão hidrófilo. Junto ao algodão foi colocado um filete de cola utilizada para captura de insetos (Bio Controle ${ }^{\circledR}$ ), para evitar a fuga dos ácaros.

O estudo foi iniciado com ovos obtidos como se segue. Inicialmente, uma fêmea de $A$. floridanus foi transferida da criação de manutenção para cada unidade de criação de $3 \mathrm{~cm}^{2}$. Após um período de seis horas, as unidades foram examinadas retirando-se as fêmeas daquelas onde havia ocorrido a oviposição, assim como os ovos excedentes, deixando-se apenas um (1) ovo por unidade. O restante das unidades foi examinado novamente após outro período de seis horas, repetindo-se o processo até que se obtivesse um total de 22 ovos. As arenas foram examinadas três vezes ao dia, às 7, 12 e 17 horas, até que os ácaros atingissem o estádio adulto, para se determinar o estádio de desenvolvimento em que o ácaro se encontrava. $\mathrm{Na}$ fase adulta, as arenas foram observadas uma vez ao dia (14 horas), para verificar o número de ovos postos.

Para se avaliar o efeito da fecundação sobre a oviposição, um teste adicional foi conduzido. Para tal, ácaros imaturos foram retirados da unidade de criação e individualizados em arenas semelhantes às descritas anteriormente.

Os ácaros foram trocados de arena uma vez por semana e em cada troca, 30 indivíduos de diferentes estádios de T. heveae foram passados para as novas arenas, para servir de alimento. Trinta fêmeas de $A$. floridanus foram utilizadas para este estudo. Quinze destas foram mantidas isoladas, enquanto que cada uma das demais foi mantida juntamente com um macho durante toda a fase adulta. Os machos foram obtidos da arena de criação, sendo substituído por novos machos, quando morriam.

As arenas foram mantidas em câmara climatizada a $25 \pm 1^{\circ} \mathrm{C}$, fotoperíodo de 12 horas e umidade relativa de $80 \pm 5 \%$.

Para verificar a significância entre as diferenças dos parâmetros médios da biologia de fêmeas adultas acasaladas e não acasaladas foi aplicado o teste t-student, considerando um intervalo de confiança de $95 \%$.

\section{Tabela de vida e fertilidade de Agistemus floridanus}

A tabela de vida foi elaborada segundo BIRCH (1948) a partir dos dados obtidos no estudo de biologia, sendo o valor de $r_{m}$ (razão intrínseca de crescimento) corrigido conforme sugerido por aquele autor.

\section{RESULTADOS}

\section{Biologia de Agistemus floridanus}

Agistemus floridanus apresentou durante seu desenvolvimento os estádios de ovo, larva, protoninfa, deutoninfa e adulto. O período de ovo-adulto durou 10,2 dias (Tab. I). O estádio de ovo foi o mais longo, com duração de 4,1 dias e os estádios de larva, protoninfa e deutoninfa duraram 1,2, 1,0 e 1,6 dias, respectivamente.

Todos os ovos das fêmeas não fecundadas originaram machos, caracterizando desta forma o desenvolvimento por partenogênese arrenótoca. As fêmeas acasaladas deram origem a machos e a fêmeas, sendo que a razão sexual foi de $85 \%$ de fêmeas.

Tabela I. Duração média, em dias ( \pm erro padrão da média) das fases imaturas e adulta e viabilidade de Agistemus floridanus alimentandose de Tenuipalpus heveae, a $25 \pm 1{ }^{\circ} \mathrm{C}$, fotofase de 12 horas e umidade relativa de $80 \pm 5 \%$.

\begin{tabular}{lcc}
\hline \multicolumn{1}{c}{ Estádios } & Duração & Viabilidade (\%) \\
\hline Ovo & $4,1 \pm 0,04$ & 100 \\
Larva & $1,2 \pm 0,1$ & 81 \\
Protocrisálida & $0,9 \pm 0,2$ & 100 \\
Protoninfa & $1,0 \pm 0,09$ & 83 \\
Deutocrisálida & $0,6 \pm 0,08$ & 100 \\
Deutoninfa & $1,6 \pm 0,2$ & 100 \\
Teliocrisálida & $0,5 \pm 0,06$ & 100 \\
Ovo-Adulto & $10,2 \pm 0,2$ & 68 \\
\hline
\end{tabular}

As fêmeas acasaladas tiveram períodos de pré-oviposição, oviposição e longevidade significativamente menores que as fêmeas não acasaladas (Tab. II). A média diária de oviposição foi significativamente maior para fêmeas acasaladas que para fêmeas não acasaladas. Para os dois grupos estudados, a oviposição total e a pós-oviposição não apresentaram diferenças estatisticamente significativas.

Tabela II. Parâmetros médios ( \pm erro padrão da média) da biologia de fêmea adultas de Agistemus floridanus acasaladas e não acasaladas, alimentando-se de Tenuipalpus heveae, a $25 \pm 1^{\circ} \mathrm{C}$, fotofase de 12 horas e umidade relativa de $80 \pm 5 \%$.

\begin{tabular}{lrc}
\hline \multicolumn{1}{c}{ Fases } & Acasaladas & Não acasaladas \\
\hline Pré-oviposição & $1,6 \pm 0,1 \mathrm{a}$ & $2,7 \pm 0,3 \mathrm{~b}$ \\
Oviposição & $16,6 \pm 1,4 \mathrm{a}$ & $23,4 \pm 3,9 \mathrm{~b}$ \\
Pós-oviposição & $7,0 \pm 1,9 \mathrm{a}$ & $6,8 \pm 1,0 \mathrm{a}$ \\
Longevidade & $25,3 \pm 2,7 \mathrm{a}$ & $34,6 \pm 4,1 \mathrm{~b}$ \\
Ovos/fêmea & $38,4 \pm 3,5 \mathrm{a}$ & $31,6 \pm 5,1 \mathrm{a}$ \\
Ovos/fêmea/dia & $2,3 \pm 0,1 \mathrm{a}$ & $1,3 \pm 0,08 \mathrm{~b}$ \\
\hline
\end{tabular}

Em cada linha, médias seguidas da mesma letra não diferem estatisticamente entre si, pelo teste $t$, a $5 \%$ de probabilidade.

\section{Tabela de vida de fertilidade}

Os resultados da análise dos dados de mortalidade e fertilidade encontram-se na tabela III. Para o cálculo da "fertilidade 
Tabela III. Tabela de vida de fertilidade de Agistemus floridanus alimentando-se de Tenuipalpus heveae. Temperatura $25 \pm 1{ }^{\circ} \mathrm{C}$, fotofase de 12 horas e umidade relativa $80 \pm 5 \%$. (x) Intervalo de idade no qual foi tomada a amostra, (Ix) taxa de sobrevivência de fêmeas no ponto médio, $(\mathrm{mx})$ fertilidade específica.

\begin{tabular}{|c|c|c|c|c|c|c|}
\hline x (dias) & $\begin{array}{l}N^{\circ} \text { de fêmeas } \\
\text { sobreviventes }\end{array}$ & Ix & $\begin{array}{l}\text { Número } \\
\text { ovos/dia }\end{array}$ & $\mathrm{mx}$ & $m x . l x$ & $m x . l x . x$ \\
\hline 0,5 & - & 1,00 & - & - & - & - \\
\hline 10,5 & 15 & 0,68 & - & - & - & - \\
\hline 11,5 & 15 & 0,68 & 8 & 0,39 & 0,26 & 3,05 \\
\hline 12,5 & 15 & 0,68 & 23 & 1,14 & 0,77 & 9,69 \\
\hline 13,5 & 15 & 0,68 & 45 & 2,25 & 1,53 & 20,66 \\
\hline 14,5 & 15 & 0,68 & 43 & 2,14 & 1,45 & 21,10 \\
\hline 15,5 & 15 & 0,68 & 49 & 2,44 & 1,65 & 25,58 \\
\hline 16,5 & 15 & 0,68 & 37 & 1,84 & 1,25 & 20,63 \\
\hline 17,5 & 15 & 0,68 & 44 & 2,19 & 1,48 & 25,90 \\
\hline 18,5 & 15 & 0,68 & 44 & 2,19 & 1,48 & 23,38 \\
\hline 19,5 & 14 & 0,63 & 38 & 2,03 & 1,27 & 24,77 \\
\hline 20,5 & 14 & 0,63 & 40 & 2,14 & 1,34 & 27,47 \\
\hline 21,5 & 13 & 0,58 & 39 & 2,25 & 1,30 & 28,06 \\
\hline 22,5 & 13 & 0,58 & 30 & 1,73 & 1,00 & 22,50 \\
\hline 23,5 & 13 & 0,58 & 31 & 1,78 & 1,03 & 24,21 \\
\hline 24,5 & 13 & 0,58 & 24 & 1,38 & 0,80 & 19,60 \\
\hline 25,5 & 12 & 0,53 & 16 & 0,99 & 0,52 & 13,26 \\
\hline 26,5 & 12 & 0,53 & 18 & 1,12 & 0,59 & 14,05 \\
\hline 27,5 & 12 & 0,53 & 19 & 1,18 & 0,62 & 17,05 \\
\hline 28,5 & 12 & 0,53 & 10 & 0,62 & 0,32 & 9,12 \\
\hline 29,5 & 12 & 0,53 & 9 & 0,56 & 0,29 & 8,56 \\
\hline 30,5 & 9 & 0,39 & 3 & 0,24 & 0,09 & 2,75 \\
\hline 31,5 & 9 & 0,39 & 2 & 0,16 & 0,06 & 1,89 \\
\hline 32,5 & 3 & 0,39 & 0 & 0,00 & 0,00 & 0,00 \\
\hline 33,5 & 6 & 0,26 & 0 & 0,00 & 0,00 & 0,00 \\
\hline 34,5 & 6 & 0,26 & 0 & 0,00 & 0,00 & 0,00 \\
\hline 35,5 & 6 & 0,29 & 0 & 0,00 & 0,00 & 0,00 \\
\hline 36,5 & 6 & 0,29 & 0 & 0,00 & 0,00 & 0,00 \\
\hline 37,5 & 6 & 0,29 & 0 & 0,00 & 0,00 & 0,00 \\
\hline 38,5 & 6 & 0,29 & 0 & 0,00 & 0,00 & 0,00 \\
\hline 39,5 & 5 & 0,24 & 0 & 0,00 & 0,00 & 0,00 \\
\hline 40,5 & 4 & 0,19 & 3 & 0,56 & 0,10 & 4,05 \\
\hline 41,5 & 4 & 0,19 & 2 & 0,37 & 0,07 & 2,91 \\
\hline 42,5 & 4 & 0,19 & 0 & 0,00 & 0,00 & 0,00 \\
\hline 43,5 & 3 & 0,14 & 0 & 0,00 & 0,00 & 0,00 \\
\hline 44,5 & 3 & 0,14 & 0 & 0,00 & 0,00 & 0,00 \\
\hline 45,5 & 3 & 0,14 & 0 & 0,00 & 0,00 & 0,00 \\
\hline 46,5 & 3 & 0,14 & 0 & 0,00 & 0,00 & 0,00 \\
\hline 47,5 & 3 & 0,14 & 0 & 0,00 & 0,00 & 0,00 \\
\hline 48,5 & 2 & 0,09 & 0 & 0,00 & 0,00 & 0,00 \\
\hline 49,5 & 2 & 0,02 & 0 & 0,00 & 0,00 & 0,00 \\
\hline 50,5 & 1 & 0,01 & 0 & 0,00 & 0,00 & 0,00 \\
\hline 51,5 & 1 & 0,01 & 0 & 0,00 & 0,00 & 0,00 \\
\hline 52,5 & 1 & 0,01 & 0 & 0,00 & 0,00 & 0,00 \\
\hline 53,5 & 1 & 0,01 & 0 & 0,00 & 0,00 & 0,00 \\
\hline 54,5 & 1 & 0,01 & 0 & 0,00 & 0,00 & 0,00 \\
\hline 55,5 & 1 & 0,01 & 0 & 0,00 & 0,00 & 0,00 \\
\hline 56,5 & 1 & 0,01 & 0 & 0,00 & 0,00 & 0,00 \\
\hline 57,5 & 0 & 0,00 & 0 & 0,00 & 0,00 & 0,00 \\
\hline$\Sigma$ & & & & 31,69 & 19,27 & 370,24 \\
\hline
\end{tabular}

específica" (mx) usou-se a razão sexual de 0,75. Observa-se que a taxa máxima de oviposição diária por fêmea foi alcançada no sexto dia (aproximadamente 2,44 ovos/fêmea/dia).

A taxa líquida de reprodução $\left(R_{o}\right)$ observada foi de 13,2 vezes a cada geração, com duração média de uma geração $(\mathrm{T})$ de cerca de 19,2 dias. A razão intrínseca de incremento populacional $\left(\mathrm{r}_{\mathrm{m}}\right)$ foi de 0,16 fêmea/fêmea/dia, enquanto a razão finita de aumento $(\lambda)$, isto é, o número de fêmeas adicionadas à população por dia, foi de 1,1 fêmea/fêmea.

\section{DISCUSSÃO}

$\mathrm{O}$ ácaro $T$. heveae demonstrou ser uma presa adequada para o desenvolvimento e a reprodução do predador $A$. floridanus. Esses resultados sugerem que uma das razões da grande abundância de $A$. floridanus, observada por Ferla \& Moraes (2002) nos clones PB 260 e PR 255 em Itiquira, seja a presença de $T$. heveae.

Entre as três espécies de ácaros predadores comuns em seringueira no Estado do Mato Grosso, Euseius concordis (Chant, 1959), Neoseiulus anonymus (Chant \& Baker, 1965) e A. floridanus, a última foi a única espécie que apresentou alta oviposição alimentando-se de C. heveae (FerLa \& Moraes 2003). Como C. heveae e T. heveae são consideradas pragas importantes desta cultura, a presença de $A$. floridanus contribui para manter os níveis populacionais destas espécies abaixo do nível de dano.

No Brasil, os estigmeídeos são ácaros predadores importantes em pomares macieira (Lorenzato 1987, Ferla \& Moraes 1998) e seringueira (Feres 2000, Ferla \& Moraes 2002). Até o momento, nenhum estudo foi desenvolvido para conhecer a tabela de vida e fertilidade destas espécies no Brasil.

Zetzellia mali (Ewing, 1917) é o Stigmaeidae mais bem conhecido, devido ao papel atribuído a esta espécie como inimigo natural de Panonychus ulmi (Koch, 1836) e Aculops schlechtendali (Nalepa, 1892) na cultura da macieira. Alimentando-se de $A$. schlechtendali, esta espécie apresentou duração média de uma geração de cerca de 21 dias e uma taxa líquida de reprodução $\left(\mathrm{R}_{0}\right)$ de 0,109 (White \& LAING 1977).

Devido à dificuldade de conseguir grandes populações de $A$. floridanus para realizar liberações inundativas, a melhor estratégia seria a liberação inoculativa em locais onde esta espécie não esteja presente, ou a definição de estratégias para conservar esta espécie nos ambientes. Até o momento, não se conhece nenhum programa que envolva a liberação inundativa com espécies da família Stigmaeidae, sendo que o principal problema é a ausência de metodologia para a criação massal de espécies desta família. Devido à utilização ocasional de agroquímicos em seringueira, é necessário a realização de estudos de seletividade de produtos em relação a este predador.

\section{AGRADECIMENTOS}

Às empresas Plantações Edouard Michellin Ltda. e Triângulo Agro-Industrial Ltda. pelo financiamento do projeto. Ao CNPq pela concessão de bolsa para a realização do doutorado do primeiro autor.

\section{REFERÊNCIAS BIBLIOGRÁFICAS}

BIRCH, L.C. 1948. The intrinsic rate of natural increase of an insect population. Journal of Animal Ecology, Cambridge,

Revista Brasileira de Zoologia 20 (2): 261-264, junho 2003 
17: $15-26$.

Ehara, S.A. 1985. New species of the genus Agistemus Summers from Hokkaido (Acarina: Stigmaeidae). Proceedengs of the Japan Academy, Tokyo, 61: 60-66.

FERES, R.J.F. 2000. Levantamento e observações naturalísticas da acarofauna (Acari, Arachnida) de seringueiras cultivadas (Hevea spp., Euphorbiaceae) no Brasil. Revista Brasileira de Zoologia, Curitiba, 17 (1): 157-173.

Ferla, N.J. \& G.J. De Moraes. 1998. Ácaros predadores em pomares de maçã no Rio Grande do Sul. Anais da Sociedade Entomológica do Brasil, Londrina, 27 (4): 649-654.

. 2002. Ácaros (Arachnida, Acari) da seringueira (Hevea brasiliensis Muell. Arg.) no Estado do Mato Grosso. Revista Brasileira de Zoologia, Curitiba, 19 (3): 867-888.

. 2003. Oviposição dos ácaros predadores Agistemus floridanus Gonzalez, Euseius concordis (Chant) e Neoseiulus anonymus (Chant \& Baker) (Acari) em resposta a diferentes tipos de alimento. Revista Brasileira de Zoologia, Curitiba, 20 (1): 153-155.

Recebido em 21.X.2002; aceito em 22.V.2003.
Gerson, U. \& R.L. Smiley. 1990. Acarine Biocontrol Agents. London, Chapman \& Hall, 174p.

LAING, J.E. \& N.F. KNoP. 1982. Potential use of predaceous mites other than Phytoseiidae for biological control of orchards pests, p. 28-35. In: M.A. Hoy; G. L. CunNingham \& L. KNuTson. Biological Control of Pests by Mites. Berkeley: University of California Press, 185p.

LoREnzATo, D. 1987. Controle biológico de ácaros fitófagos na cultura da macieira no município de Farroupilha, RS. Agronomia Sulriograndense, Porto Alegre, 23 (2): 167-183.

McMurtry, J.A.; C.B. Huffaker \& M. van de VRIE. 1970. Ecology of tetranychid mites and their natural enemies: a review. 1. Tetranychid enemies: Their biological characters and the impact of spray practices. Hilgardia, Berkeley, 40 (11): 331390.

White, N.D. \& J.E. Laing. 1977. Some aspects of the biology and a laboratory life table of the acarine predator Zetzellia mali. The Canadian Entomologist, Ottawa, 109: 12751281. 